\title{
Editorial
}

\section{Atracurium and vecuronium}

Muscle relaxation, using curare, was introduced into anaesthetic practice by Griffith and Johnson in Montreal in 1942. ${ }^{1}$ Thus, it is ironic that the new neuromuscular blocking drugs, atracurium and vecuronium, have been released in Canada later than in most other Western countries. Nonetheless, they have arrived and the delay has given us the opportunity to reexamine what we need.

Our demands have not altered since 1942. We use muscle relaxants to provide good conditions for endotracheal intubation and to produce abdominal muscle relaxation to facilitate surgery. But, the requirements for these tasks are not identical. For intubation, we require rapid, intense paralysis of short duration, whereas, for maintenance, longer lasting but less complete relaxation is necessary. Here, an ability to produce recovery at the end of surgery is more important. Clearly, absence of side effects and of unwanted actions are advantageous. Indeed, some of the impetus to replace the existing agents was to avoid the often severe problems associated with succinylcholine and the cardiovascular sequelae of d-tubocurarine and pancuronium.

Atracurium and vecuronium are powerful, nondepolarizing muscle relaxants. Their potencies may be expressed as the doses required to produce 95 per cent depression of the thumb twitch tension in response to stimulation of the ulnar nerve. This $E D_{95}$, or multiples or fractions of it, is a useful index in describing dosage. During balanced, $\mathrm{N}_{2} \mathrm{O}$ $\mathrm{O}_{2}$-narcotic anaesthesia the $\mathrm{ED}_{95}$ for atracurium is $0.2 \mathrm{mg} \cdot \mathrm{kg}^{-1}$, and for vecuronium $0.05 \mathrm{mg} \cdot \mathrm{kg}^{-1}$ compared with $0.5 \mathrm{mg} \cdot \mathrm{kg}^{-1}$ for d-tubocurarine and $0.08 \mathrm{mg} \cdot \mathrm{kg}^{-1}$ for pancuronium. The onset of paralysis using equipotent doses of atracurium or

From the Department of Anaesthesia, Royal Victoria Hospital and McGill University, 687 Pine Avenue West, Montreal, Quebec H3A 1 Al. vecuronium is only slightly more rapid than with pancuronium but their durations of action are only one third as long. The rapid recovery is a result of spontaneous degradation (Hofmann elimination) for atracurium and increased plasma clearance for vecuronium. Their outstanding advantage is that, in clinical doses, they are virtually free of cardiovascular effects. ${ }^{2}$

This freedom from cardiovascular complications will make them attractive to anaesthetists. However, to obtain satisfaction clinicians will need to modify their anaesthetic techniques. Initial doses of 2-3 $\times E_{95}$ have been recommended before intubation. This corresponds to doses of $30-40 \mathrm{mg} /$ $70 \mathrm{~kg}$ for atracurium and $7-10 \mathrm{mg} / 70 \mathrm{~kg}$ for vecuronium. Even with these large doses good intubating conditions will not be produced as rapidly as with succinylcholine, $1 \mathrm{mg} \cdot \mathrm{kg}^{-1}$. Increasing the dose or administering it in divided doses (priming principle ${ }^{3}$ ) will produce some acceleration but most anaesthetists will simply wait longer for the drugs to act. Similarly, for the maintenance of relaxation repeated doses of $\frac{1}{4}-\frac{1}{2} \times E_{95}$ will lead to very frequent dosing. Some have tried a continuous infusion of $\mathrm{ED}_{95} \times 1.5 /$ hour following an initial bolus of $1 \times E_{95}{ }^{4}$ This will require careful neuromuscular monitoring and recovery is slower after stopping the infusion than after bolus injection. Recovery, of course, can be accelerated with anticholinesterases and edrophonium would appear to be a good choice. ${ }^{5,6}$

Many situations will prove ideal for the new agents. They can be substituted for succinylcholine in short procedures and in conditions where the traditional drugs are hazardous, e.g., malignant hyperthermia, renal failure. Atracurium has been used in myasthenia gravis ${ }^{7}$ taking advantage of its metabolism which allows recovery of neuromuscular function without the use of anticholinesterases. For surgery of longer duration or in the intensive 
care unit their place and method of use are unclear. Several other questions remain: is reversal always necessary and, if so, which antagonist is best? Can the onset time be reduced safely? Are the metabolites of either drug toxic? No doubt, clinical anaesthetists will answer these questions rapidly. The basic pharmacology of the agents is well known but the best way to use them will require great ingenuity.

\section{Atracurium et vécuronium}

Le relâchement musculaire, par le curare, a été introduit dans la pratique anesthésique par Griffith et Johnson à Montréal en 1942.' Ainsi il paraît ironique que les nouveaux bloqueurs neuromusculaires, atracurium et vécuronium, soient mis sur le marché canadien après leur introduction dans la majorité des pays de l'ouest. Ils sont néanmoins arrivés et le délai nous a donné l'opportunité de les étudier en fonction de nos besoins.

Nos demandes n'ont pas été altérées depuis 1942. On utilise les relaxants musculaires afin de fournir des conditions adéquates pour l'intubation endotrachéale et maintenir un relâchement musculaire pouvant faciliter la chirurgie. Cependant les requis pour ces tâches ne sont pas identiques. Pour l'intubation on demande une paralysie intense, rapide et de courte durée. Pour le maintien, une relaxation musculaire moins complète mais de plus longue durée est nécessaire. Aussi, pour cette dernière tâche la capacité de provoquer une reprise de la fonction musculaire à la fin de la chirurgie est importante. Il est aussi évident que l'absence d'effets secondaires ou d'effets non désirables représenterait un avantage. En effet, une des raisons de remplacer les agents existants était d'éviter les problèmes souvent sévères associés à la succinylcholine ou les séquelles cardiovasculaires de la d-tubocurarine et du pancuronium.

L'atracurium et le vécuronium sont de puissants bloqueurs neuromusculaires non dépolarisants. Leur puissance peut être exprimée comme étant la dose requise pour produire 95 pour cent de dépres- sion de la tension de contraction du pouce en réponse à une stimulation du nerf cubital. Cet $\mathrm{ED}_{95}$, ses multiples ou ses fractions, est un index utile pour l'étude des doses. Lors d'une anesthésie balancée au protoxyde-oxygène et narcotiques, le $E D_{95}$ de l'atracurium est de $0.2 \mathrm{mg} \cdot \mathrm{kg}^{-1}$, et celui du vécuronium de $0.05 \mathrm{mg} \cdot \mathrm{kg}^{-1}$ comparativement à $0.5 \mathrm{mg} \cdot \mathrm{kg}^{-1}$ pour la d-tubocurarine et $0.08 \mathrm{mg} \cdot \mathrm{kg}^{-1}$ pour le pancuronium. Le début de la paralysie lors l'utilisation de doses équipotentes d'atracurium ou de vécuronium est légèrement plus rapide qu'avec le pancuronium. Cependant leur durée d'action n'est que le tiers de celle du pancuronium. La reprise rapide de la fonction neuromusculaire est le résultat de la dégradation spontanée (élimination de Hofmann) pour l'atracurium et la clairance plasmatique augmentée pour le vécuronium. Leur avantage exceptionnel demeure le fait qu'à des doses cliniques, ils sont pratiquement libres d'effets cardiovasculaires. $^{2}$

Cette absence de complication cardiovasculaire les rendra attrayants aux anesthésistes. Cependant, afin d'obtenir satisfaction, les cliniciens doivent modifier leurs techniques anesthésiques. Les doses initiales de deux à trois fois le $E D_{95}$ ont été recommandées avant l'intubation. Ceci correspond à des doses de 30 à $40 \mathrm{mg} / 70 \mathrm{~kg}$ pour l'atracurium et $7 \mathrm{a} 10 \mathrm{mg} / 70 \mathrm{~kg}$ pour le vécuronium. Même avec ces doses élevées, les conditions idéales d'intubation ne seront pas reproduites aussi rapidement qu'avec la succinylcholine $1 \mathrm{mg} \cdot \mathrm{kg}^{-1}$. L'augmentation de la dose ou son administration à petites doses (priming principles ${ }^{3}$ ) produira une légère accélération mais la majorité des anesthésistes attendront tout simplement plus longtemps pour que les drogues fassent effet. De même pour le maintien de la relaxation, des doses répétées de $\frac{1}{4}$ à $\frac{1}{2}$ de la $\mathrm{ED}_{95}$ aboutiront à des répétitions fréquentes des doses. Certains ont essayé la perfusion continue de $1.5 \times$ la $\mathrm{ED}_{95}$ par heure après l'administration du bolus initial de $1 \times$ le $\mathrm{ED}_{95} .{ }^{4}$ Ceci demandera une surveillance accrue de la fonction neuromusculaire. On observera aussi une reprise plus lente de la fonction neuromusculaire comparativement à la technique du bolus. Cette reprise de la fonction neuromusculaire pourra certainement être accélérée par l'administration d'antichlinestérasiques et l'édrophonium apparaît comme étant un bon choix. . $^{5,6}$

Plusieurs situations se révèleront comme étant 
idéales pour l'emploi des nouveaux agents. Ils peuvent remplacer la succinylcholine pour les courtes procédures et dans les conditions où l'emploi des drogues traditionnelles est hasardeux, e.g. hyperthermie maligne ou insuffisance rénale. L'atracurium a été utilisé dans la myasthénie grave à cause de l'avantage qu'il procure par son métabolisme permettant une reprise de la fonction neuromusculaire sans l'emploi d'anticholinestérasiques. Pour la chirurgie de longue durée, ou dans les soins intensifs, leur place ainsi que la méthode d'administration reste à éclaircir. Plusieurs autres questions demeurent: est-ce que l'antagonisme est toujours nécessaire et si oui, quel antagoniste serait le meilleur? Est-ce que le début d'action peut être réduit avec sécurité? Est-ce que les métabolites de ces deux médicaments sont toxiques? Sans aucun doute les réponses à ces questions viendront rapidement avec l'emploi de ces médicaments. La pharmacologie de base de ces agents est bien connue mais la meilleure façon de les utiliser demandera une grande ingénuosité.

\section{References}

1 Griffith HR, Johnson GE. The use of curare in general anesthesia. Anesthesiology 1942; 3:418-20.

2 Miller RD, Rupp SM, Fisher DM, Cronnelly $R$, Fahey $M R$, Sohn $Y J$. Clinical pharmacology of vecuronium and atracurium. Ancsthesiology 1984; 61: 444-53.

3 Doherty WG, Breen PJ, Donati F, Bevan DR. Accelerated onset of pancuronium with divided doses. Can Anaesth Soc J 1985; 32: 1-4.

4 Noeldge $G$, Hinksen $H$, Buzello W. Comparison between the continuous infusion of vecuronium and the intermittent administration of pancuronium and vecuronium. Br J Anaesth 1984; 56: 473-7.

5 Jones RM, Pearce AC. Williams JP. Recovery characteristics following antagonism of atracurium with neostigmine or edrophonium. Br J Anaesth 1984; 56: 453-7.

6 Baird WLM, Bowman WC, Kerr WJ. Some actions of ORG NC45 and of edrophonium in the anaesthetized cat and in man. Br J Anaesth 1982; 54: 375-85.

7 Baraka A, Dajani A. Atracurium in myasthenics undergoing thymectomy. Anesth Analg 1984; 63 : $1127-30$. 